11 - ORIGINAL ARTICLE

STATE OF ART

\title{
To proliferate, not to proliferate or to die. The liver itself decides as needed
}

Orlando de Castro e Silva ${ }^{\mathrm{I}}$, Marina Rodrigues Garcia da Silveira ${ }^{\mathrm{II}}$

IPhD, Full Professor, Head, Department of Surgery and Anatomy, Ribeirao Preto School of Medicine, Sao Paulo University (USP), Ribeirao Preto-SP, Brazil. Conception, design, intellectual and scientific content of the study, manuscript writing.

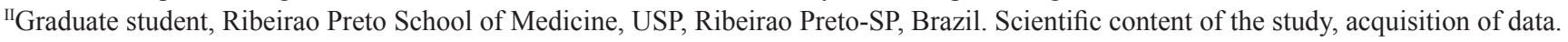


The liver has an extraordinary capacity for regeneration in the presence of various types of challenges or injuries, including acute hepatitis and partial liver resection ${ }^{1-3}$. The tissue loss rapidly triggers the regenerative process until the original weight is recovered $^{1,2,4}$. The term hepatic regeneration, although commonly used, is biologically incorrect since the response induced by the resection of hepatic tissue is not truly regenerative. The resected lobes do not develop again. The restoration of hepatic mass occurs by compensatory cell hyperplasia in the remaining lobes, with a consequent increase in its dimensions ${ }^{5-7}$. This suggests that hepatic growth may be controlled by functional rather than anatomical factors. Whatever the nature of these factors, they seem to be quite precise since growth ceases when the liver reaches its original weight. Hepatocyte proliferation does not become dysregulated even after consecutive resections. Simpson and Finckh $^{7}$ observed complete restoration of the hepatic mass after a series of five successive hepatectomies at five to seven week intervals ${ }^{7}$. After the first partial hepatectomy $(\mathrm{PH})$, regeneration occurred by means of an increased number and size of hepatic lobules, whereas after the subsequent hepatectomies there was a progressive predominance of increased numbers of hepatic lobules ${ }^{1,6-8}$ (Figure 1).

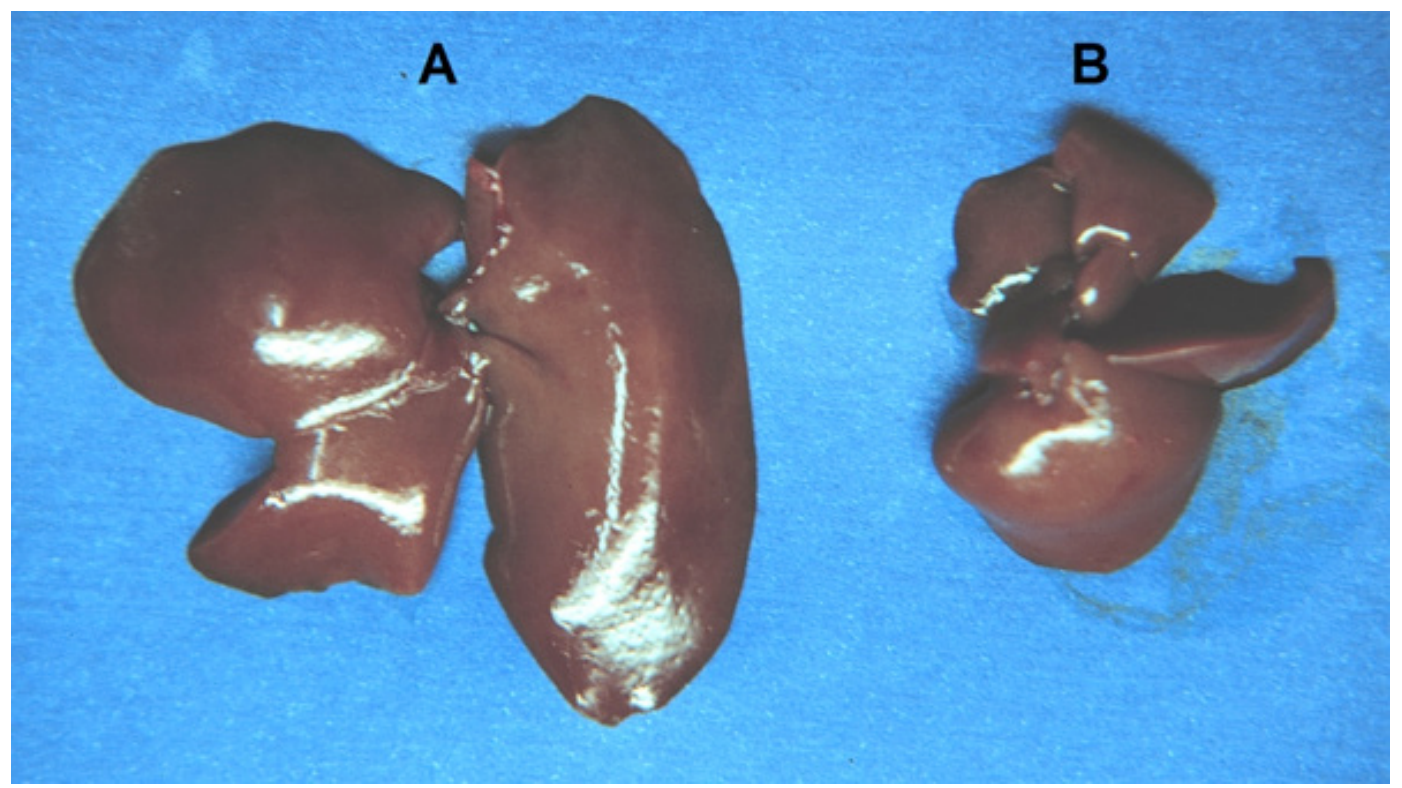

FIGURE 1 - Rat liver consisting of the left and middle lateral lobes (A) and remnant liver-RL-right and caudate lobes (B) corresponding to approximately 70 and $30 \%$ of the total hepatic mass, respectively ${ }^{8}$.

After $\mathrm{PH}$, the remnant liver (figure 1) exhibits a regenerative response, always maintaining a percent relation with the host, with the RL receiving through the portal vein the hepatotropic factors responsible for hepatic tropism in the presence of portal hypertension. After PH, portal hypertension occurs both due to increased flow to the RL and to the increased resistance of the RL to portal flow ${ }^{9-12}$.

After PH, the RL needs to adapt hemodynamically since, after a $70 \% \mathrm{PH}$, for example, the remaining 30\% part of the organ will receive the entire arterial and portal flow that used to irrigate the whole organ before $\mathrm{PH}$. The increase in portal flow in the presence of an additional regimen of portal hypertension, which is proportional to the size of the RL (the smaller the RL, the higher the increase), induces a reduction of hepatic artery flow which may impair the hemodynamic adaptation of the RL but not its regeneration. This causes a metabolic overload in the RL that seems to trigger the process of liver regeneration. Studies of hepatic blood flow have shown that total or partial portocaval shunts lead to RL hypotrophy or even atrophy, delaying or impairing hepatic regeneration. A series of experiments have clearly shown the dependence of hepatic tissue on portal blood, especially regarding hormones, nutrients, genes and growth factors originating from the splanchnic territory ${ }^{8-12}$.

Hepatic growth factors include a large number of substances that can be divided into three categories: 1. Mitogenic agents (e.g. HGF, EGF); 2. Co-mitogenic agents (e.g. TNFa, IL-6, norepinephrine, insulin, vasopressin, angiotensin I and II, estrogen, testosterone); 3. Inhibitory agents (interleukin 1 and growth transforming factor beta $(\beta T G F))$. The main concept arising is that hepatic regeneration is a process involving various steps with at least two critical points, i.e., the transition from the quiescent phase (G0 to G1) of the hepatocyte to the cell cycle (priming or gene activation), 
progression from the restriction point in the G1 phase of the cycle, and progression to DNA synthesis (S phase). The hepatocytes must be primed in order to become competent and to respond to growth factors since they are dormant in the G0 phase. This priming is a reversible process that is not self-sufficient for replication, performed by cytokines and hormonal and nutritional signals $\mathbf{s}^{1,2,4,5,11,12}$.

It has been proposed that these steps may be under separate types of control, i.e., priming by the cytokines tumor necrosis factor alpha (TNF $\alpha$ ) and interleukin 6 (IL-6) and control of the cell cycle by the hepatocyte growth factor (HGF) and by transforming growth factor alpha $(\mathrm{TGF} \alpha)^{1,9,10}$. As stated earlier, the progression of the cell cycle requires growth factors, but the hepatocytes need to be sensitized $d^{1,2,4,5,11,12}$.

Gene expression in hepatic regeneration can be divided into various phases starting with the expression of a large number of "immediate" genes. The proto-oncogenes $c$-fos, $c$-jun and $c$-myc have been identified, but more than 70 genes have been found to be involved, although we still do not know how to determine the importance of each. Transcription factors are proteins whose specific sites of recognition are located in genes in order to start their transactivation, so that they can propagate signals by activation of various different genes. At least four transcription factors play a major role in the initiation of hepatic regeneration (NFkB, STAT3, AP-1 and C/EBP $\beta)^{1,2,4,5,11,12}$.

The expression of RH genes does not lead to DNA replication unless the cells advance through the cell cycle. This is achieved by genes such as HGF and TGF $\alpha$. Once the hepatocytes go through this restriction point and express cyclin D1, the cell is irreversible led to replication. Cyclin D1 is the most important marker for the progression of the cell cycle in the hepatocytes (G1 phase). Cyclin D1 has two peaks that coincide with the two waves of DNA replication after $\mathrm{PH}$ in rats, and its expression probably establishes the stage where replication becomes autonomous. The expression of cyclin D1 signals the G1/S point, where the cells progress to replication independent of growth factors ${ }^{1,2,4,5,11,12}$.

In the second phase of gene expression after $\mathrm{PH}$, the transcription of these genes is blocked by inhibitors of protein synthesis, an example being $\mathrm{Bcl}-\mathrm{Xl}$, which is the principal antiapoptotic gene expressed in the liver. It has not been determined whether this expressed gene prevents apoptosis in early stages of hepatic regeneration or if it has other roles. A possibility is that it may function as an antioxidant agent in order to prevent damage induced by the reactive oxygen species generated by the mitochondria. The equilibrium between stimulators and inhibitors expressed after hepatectomy can explain why hepatic regeneration is a firmly regulated growth process ${ }^{1,2,4,5,11-13}$.
The liver can regulate its growth and mass in a precise manner according to the stimuli it receives. As stated earlier, the ability of the liver to respond or to adapt to variable metabolic necessities is intact. The surgical resection of hepatic lobes, as well as the loss of parenchyma provoked by a virus or by chemical injury triggers hepatocyte replication, although in a controlled process involving simultaneous apoptosis. This particular occurrence is observed in the presence of deficit or excess of hepatic mass, the latter represented by the so-called large for size liver transplant in which the capacity of the received liver exceeds the metabolic demand of the recipient and controlled liver remodeling occurs ${ }^{1,2,4,5,11-13}$.

There are many conditions in which changes in hepatic mass (increase or reduction) are present. The deficits frequently originate from the surgical removal of tissue and from cell loss (functional deficit without necessarily involving a loss of mass) caused by chemical agents or viruses. Lobe or segmental resections are performed in order to remove tumors or to obtain a transplant from a living donor. The transplantation of a small liver to a large recipient ("small for size transplant") is a current example of functional hepatic deficiency ${ }^{9-11}$. Other examples of functional deficiency involve cellular and tissue necrosis with no changes in mass, such as massive necrosis in acute liver failure and the extensive cell death caused by toxic agents and viruses.

The decisive point for the regulation of hepatic growth is due more to the ratio between the mass of the organ and the body mass than to the hepatic mass per se. The optimization of the ratio indicates that the liver reaches a state in which it performs the amount of metabolic work necessary to satisfy the requirements of the organism. The regulation of the hepatic mass does not simply occur due to growth and when the mass exceeds the functional metabolic demand, the liver loses mass in order to restore the appropriate liver/body ratio by means of hepatocyte apoptosis. In a situation in which the capacity of the liver exceeds the needs of the recipient, the hepatic mass decreases until reaching the most appropriate point of equilibrium ${ }^{1,2,4,5,11,12}$. This shows that the process of hepatocellular liver regeneration depends on the liver/host ratio. It is also known that primary hepatocytes rarely proliferate in vitro; however, in the presence of growth factors hepatocyte proliferation can be induced. We did not detect any investigations of ex-situ hepatic regeneration under conditions of hypothermal preservation after partial hepatectomy, a situation in which the RL loses its bodily and hemodynamic relationship with the host and is kept under conditions favorable to the maintenance of its tissue, physiological and biochemical vitality ${ }^{14-16}$, although theoretically adverse to RL regeneration. The Figure 2 shows the sequence of events, from partial hepatectomy $(\mathrm{PH})$ until liver regeneration. 


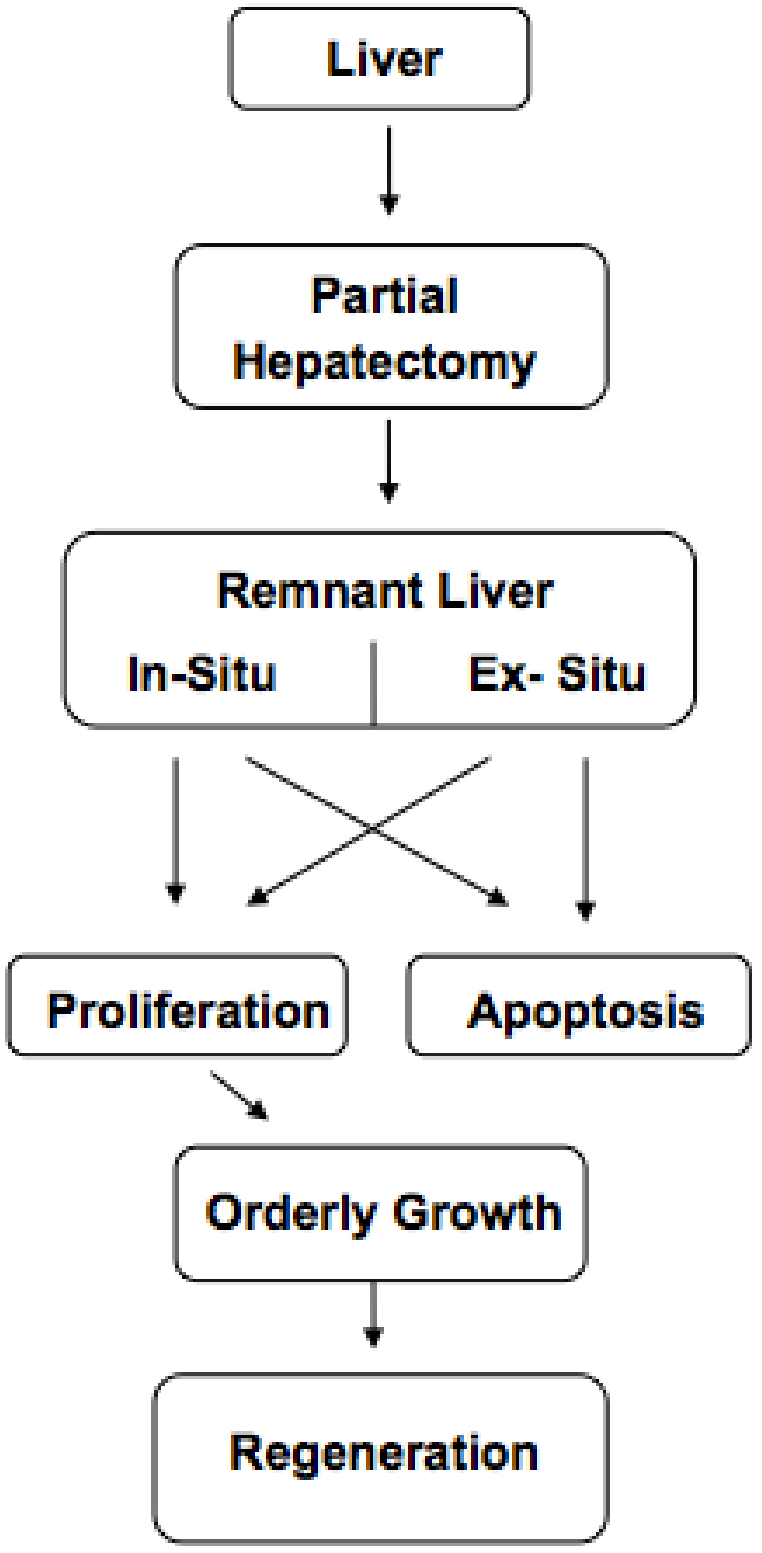

FIGURE 2 - Sequence of events from partial hepatectomy (PH) until liver regeneration.

However, preliminary studies from our laboratory have demonstrated incipient regeneration of $\mathrm{RL}$ hypothermically preserved under adverse conditions between 0 and $4^{\circ} \mathrm{C}$, suggesting that the liver can grow under conditions that do not necessarily obey its relation with body mass currently described in the literature. In that investigation, presented at the VII Brazilian Congress of Liver, Pancreas and Small Bowel, Brazil 2012, where it received an award, and published in summarized form in the journal Medicina, Ribeirão Preto $^{17}$, the RL resulted from partial ex-situ $70 \%$ hepatectomy and was maintained in hypothermal preservation medium for 24 hours. Thus, the RL was maintained outside the animal's body, receiving no information from it that might trigger or maintain hepatocellular growth. In pressure terms, the portal system of the RL did not progress to portal hypertension, as is usually the case after partial hepatectomies with small RL due to the reduction of hepatic mass and the increase in mainly portal flow. And, under these conditions, the RL showed significant cell division. This finding may signal new paths for the study of liver regeneration.

\section{References}

1. Michalopoulos G. Liver regeneration: molecular mechanisms of growth control. FASEB J. 1990;4:176-87.

2. Fausto N. Liver regeneration. J Hepatol. 2000;32:19-31.

3. Castro e Silva O, Silveira MRG. Fígado e luz. Uma combinação precisa, da mitologia à medicina. Medicina (Ribeirão Preto). 2013;46(3):318-21.

4. Mortensen KE, Revhaug A. Liver regeneration in surgical animal models - A historical perspective and clinical implications. Eur Surg Res. 2011;46:1-18.

5. Miyaoka Y, Miyajima A. To divide or not to divide: revisiting liver regeneration. Cell Div. 2013;8:1-12.

6. Yuan Q, Loya K, Rani B, Mobus S, Balakrishnan A, Lamle J, Cathomen T, Vogel A, Manns MP, Ott M, Cantz T, Sharma AD. MicroRNA-221 overexpression accelerates hepatocyte proliferation during liver regeneration. Hepatology. 2013;57:299-310.

7. Simpson GE, Finckh ES. Pattern of regeneration of rat liver after repeated partial hepatectomies. J Pathol Bacteriol. 1963;86:361-70.

8. Higgins GM, Anderson RM. Experimental pathology of the liver: I. Restoration of the liver of the white rat following partial surgical removal. Arch Pathol. 1931;12:186-202.

9. Asencio JM, Vaquero J, Olmedilla L, García Sabrido JL. "Small-for-flow" syndrome: shifting the "size" paradigm. Med Hypotheses. 2013;80:573-7.

10. Leng J, Xing H, Tan J, Chen K, Dong J. The safe minimally ischemic liver remnant for small-for-size syndrome in porcine hepatectomy. Transplant Proc. 2013;45:2419-24.

11. Mori S, Kim H, Park MS, Choi Y, Hong G, Yi NJ, Lee KW, Suh KS. Graft regeneration rate and small-for-size syndrome in living donor liver transplantation. Hepatogastroenterology. 2013;60:1463-8.

12. Fausto N, Mead JE. Regulation of liver growth: protooncogenes and transformin growth factors. Lab Invest. 1989;60:4-13.

13. Castro e Silva T, Castro e Silva O, Kurachi C, Ferreira J, Zucoloto $\mathrm{S}$, Bagnato VS. The use of light-emitting diodes to stimulate mitochondrial function and liver regeneration of partially hepatectomized rats. Braz J Med Biol Res. 2007;40:1065-9.

14. Paddenberg R, Howold N, Hoger C, Janssen H, Grau V, Kummer W. Organ preservation solutions attenuate accumulation and nuclear translocation of hypoxia-inducible factor-1alpha in the hepatoma cell line HepG2. Cell Biochem Funct 2009;27:516-25.

15. Fuller BJ, Petrenko AY, Rodriguez JV, Somov AY, Balaban CL, Guibert EE. Biopreservation of hepatocytes: current concepts on hypothermic preservation, cryopreservation, and vitrification. Cryo Letters. 2013;34:432-52.

16. Lu YC, Untaroiu CD. Effect of storage methods on indentationbased material properties of abdominal organs. Proc Inst Mech Eng H. 2013;227:293-301.

17. Silveira M, Figueiredo D, Silva A, Castro e Silva O. Liver regeneration and mitochondrial function after partial hepatectomy in ex-situ hypothermically preserved livers for 24 hours. Medicina (Ribeirão Preto). 2012;45(4):13. 
Correspondence:

Orlando de Castro e Silva Jr

Departamento de Cirurgia e Anatomia

Faculdade de Medicina de Ribeirão Preto, USP

Campus Universitário

14049-990 Ribeirão Preto - SP Brasil

Tel.: (55 16)3632-4233

orlando@fmrp.usp.br 